\title{
A hippocampus-inspired illumination time-resolved device for neural coding
}

\author{
Caihong $\mathrm{Li}^{1}$, Wen $\mathrm{Du}^{1}$, Hezhuang $\mathrm{Liu}^{1}$, Mao $\mathrm{Yang}^{2}$, Hao $\mathrm{Xu}^{2,3^{*}}$, Jiang $\mathrm{Wu}^{1,3^{*}}$ and Zhiming Wang ${ }^{1^{*}}$
}

\begin{abstract}
Artificial photonic synapses have set off a new upsurge for mimicking a series of neural activities in recent years. In particular, the investigation of learning and memory behaviors with pressure or emotion and corresponding mechanisms is currently the focus of more attention. Herein, a hippocampus-inspired device based on $\mathrm{MoS}_{2}$ for illumination time encoding is fabricated, in which the encryption technology is employed for data security. In addition, the pressureinduced memory behaviors with full memory function (memory trace) over time such as encoding, storage and retrieval are demonstrated, resulting from the decreasing positive photocurrent of the $\mathrm{MoS}_{2}$ devices. The proposed mechanism of the memory effect when exposed to the light is elucidated in detail. Moreover, the effect of stress hormone on memory behavior is displayed via different illumination time periods and light intensities. These results indicate the potential application of $\mathrm{MoS}_{2}$ devices in artificial neural network.
\end{abstract}

Keywords: illumination time, memory trace, pressure-induced memory behavior, memory encoding, memory retrieval

\section{INTRODUCTION}

Neural coding aims to reveal the stimuli-response function where the stimuli and the response mean what and how is being encoded, respectively [1]. Notably, neural decoding, i.e., figuring out how neurons intercommunicate, is a significant step to understand how nervous systems operate [2]. Generally, the major categories of neural coding include rate code and temporal code [3]. For instance, Gupta et al. [4] demonstrated a neural spike encoder using a memristor-based neural activity sensor. In terms of temporal code, learning can be understood as activity-dependent synaptic delay modifications [5]. Unlocking the process of how neurons work, especially encoding and decoding, is of importance to the future development of artificial intelligence (AI) [6]. Given this, researchers have been attempting to find out the relationship between neurons and actions to decipher memory traces of brain [6]. Richard Semon, a German memory theorist, introduced the concept of "engram" in 1904 which is roughly equivalent to the contemporary term of "memory trace" [7]. As studied, the memory trace has been divided into three independent stages, including encoding, storage and retrieval [8]. Memory encoding means the initial experience of receiving information from sensory input in the forms of stimulus, while memory storage and retrieval refer to retention and recall of information over time, respectively [8].

Recently, optically stimulated synaptic devices are well-known for wide bandwidth, low power consumption, fast signal propagation speed and efficient interconnect, which contribute to neuromorphic computing and mimicking neural activities [913]. For example, optically stimulated synaptic devices have been applied to mimic the memory storage of the brain, such as short term memory (STM) and long term memory (LTM) which are two important components of the Atkinson-Shiffrin memory model $[9,14,15]$. Nevertheless, the full memory function has been rarely reported, and the proposed mechanisms have remained unknown. Especially, the pressure-induced memory behavior $[16,17]$ is a subject of growing concerns, because the pressure can lead to brain aging in advance revealed by some Alzheimer's experts [18]. Thereupon, the study regarding illumination time-resolved memory properties and tracing full memory behaviors is of great worthy. Varied information can be carried by different temporal patterns such as illumination time and/or interspike intervals (ISI), and the latter is considered as the simplest temporal code [19].

Currently, two-dimensional (2D) materials have been widely fabricated to optically modulate artificial synapses due to their excellent photoresponse, well-developed synthesis methodology and potential integration with complementary metal oxide semiconductor (CMOS) technologies [14,20-27]. For example, Mennel et al. [21] have reported a neural network image-senor array [28] based on $\mathrm{WSe}_{2}$ for ultrafast image recognition and encoding. Furthermore, the memory effect, a defect-induced persistent photoconductivity (PPC) effect in 2D materials $[29,30]$, has opened up potential applications towards optical neural network [31], since it is similar to the memory effect of brain. These defects can trap charge carriers for a long moment while the detrapping process of charge carriers can be extremely slow [32]. The well-accepted trapping/detrapping processes can be caused by crystal defects, semiconductor/dielectric interfaces and heterojunctions [9,33-37]. Park's group [13] verified that the PPC effect in $\mathrm{ReS}_{2}$ resulted from sulphur vacancies via density functional theory (DFT) calculations. Furthermore, scanning tunneling spectroscopy and X-ray photoelectron spectroscopy confirmed the dangling bonds [12] and interfaces [38] had introduced the PPC effect, respectively. Benefitted from high optical absorption $[24,33,34]$, broadband photodetection [39-41], designed and controllable charge trapping [23] and

\footnotetext{
${ }^{1}$ Institute of Fundamental and Frontier Sciences, University of Electronic Science and Technology of China, Chengdu 610054, China

${ }^{2}$ School of Physics, University of Electronic Science and Technology of China, Chengdu 610054, China

${ }^{3}$ State Key Laboratory of Electronic Thin Films and Integrated Devices, University of Electronic Science and Technology of China, Chengdu 610054, China

* Corresponding authors (emails: hao.xu.15@uestc.edu.cn (Xu H); jiangwu@uestc.edu.cn (Wu J); zhmwang@uestc.edu.cn (Wang Z))
} 
thus the inherent PPC effect [42,43], molybdenum disulfide $\left(\mathrm{MoS}_{2}\right)$ can be a promising candidate for photonic or optoelectronic synapses as the photosensitive layer [39,44]. Jiang's group [45-49] have performed plentiful intriguing studies about $\mathrm{MoS}_{2}$-based optoelectronic synapses. Based on trappingdetrapping mechanisms, they can achieve the mimicking of rich and complicated synaptic functions such as stimulating classical Pavlovian condition, spike rate coding, and visual adaptation [45-47]. Up to now, encoding the information of illumination time by the positive/negative PPC has been rarely studied.

In this work, we have fabricated an illumination time-resolved memory device based on $\mathrm{MoS}_{2}$ for neural coding. This twoterminal device demonstrates the pressure-induced full memory trace including encoding, storage and retrieval. The proposed mechanism of decreasing positive current with illumination time is presented. This research opens a new way to encode the illumination time in the memory trace and shows the prospects in neural coding.

\section{EXPERIMENTAL SECTION}

The low-pressure chemical vapor deposition (LPCVD)-grown few-layer $\mathrm{MoS}_{2}$ was prepared by the one-zone tube furnace (Lindberg/Blue M, TF55035KC-1) with a one-inch quartz tube. $\mathrm{NaCl}$ (1.3 mg) was mixed with $8.5 \mathrm{mg} \mathrm{MoO}_{3}$ as the Mo precursor and excess sulfur powder were placed downstream and upstream of the tube, respectively. The distance between Mo and $\mathrm{S}$ precursors were about $23 \mathrm{~cm}$. The $\mathrm{Si} / \mathrm{SiO}_{2}$ substrate was cleaned by acetone, isopropanol and deionized water of $10 \mathrm{~min}$, respectively, followed by drying under nitrogen. Oxygen plasmatreated (PLUTOVAC, Pluto-T, $180 \mathrm{~W}, 180 \mathrm{~s}$ ) substrates were placed in pair in the quartz boat and the vertical distance between the substrates was $0.2 \mathrm{~cm}$. The pressure of the tube was kept at about $50 \mathrm{~Pa}$ with $40 / 5 \mathrm{~mL} \mathrm{~min}^{-1}$ of $\mathrm{Ar} / \mathrm{H}_{2}$ carrier gas after $10 \mathrm{~min}$ of vacuum-pumping. The furnace center was heated up to $750^{\circ} \mathrm{C}$ for $\mathrm{MoS}_{2}$ growth and at the same time the tem- perature of sulfur powder was ramped up to $220^{\circ} \mathrm{C}$. The $\mathrm{MoS}_{2}$ growth temperature was maintained for half an hour and then the furnace was cooled to room temperature. Indium was deposited on $\mathrm{MoS}_{2}$ as the source and drain electrodes [50,51].

\section{RESULTS AND DISCUSSION}

Generally, the CVD-grown $\mathrm{MoS}_{2}$ films show inevitable defects and impurities, which possess either beneficial or detrimental effects on targeted applications [52-54]. The defects can degrade carrier mobilities as well as prolong the lifetime of photogenerated carriers [52]. For example, defects can result in enhancement of quantum efficiency with the long-lived trap states [55]. Fig. 1a shows the optical image of an as-grown meshed $\mathrm{MoS}_{2}$ sample with multilayer flakes on the continuous film prepared by LPCVD [56]. Due to the obvious optical contrast, the multilayer domains (bluish) and underlying film (pinkish) can be easily distinguished. In Fig. 1b, the typical Raman spectra of layered $\mathrm{MoS}_{2}$ are depicted, with no characteristic peak of bulk ones resolved around $284 \mathrm{~cm}^{-1}$ [57]. The frequency differences between the $\mathrm{E}_{2 \mathrm{~g}}$ and $\mathrm{A}_{1 \mathrm{~g}}$ modes are about 22 and $25 \mathrm{~cm}^{-1}$ of marked $\mathrm{P} 1$ and $\mathrm{P} 2$ spots in Fig. 1a, respectively, consistent with the previously reported few-layer [58] and multilayer [58] $\mathrm{MoS}_{2}$ films. In addition, the weak and broad peak near $450 \mathrm{~cm}^{-1}$, namely, $2 \mathrm{LA}(\mathrm{M})$, can be attributed to the double resonance effect under the $532 \mathrm{~nm}$ laser excitation [59]. In addition, the corresponding Raman mapping result of $\mathrm{MoS}_{2}$ from a randomly selected area of $14 \mu \mathrm{m} \times 14 \mu \mathrm{m}$ is shown in Fig. 1c. The distinct difference of Raman intensity via color contrast in the mapping image further confirms a mix of thicker (green) and thinner (red) regions of $\mathrm{MoS}_{2}$. The overlapped multilayer domains suggest a potential mode of the $\mathrm{MoS}_{2}$ film growth following the mechanism of Ostwald ripening [60,61]. Fig. 1d illustrates the photoluminescence (PL) spectrum with the broad exciton A and B peaks, originated from the spin-orbital interaction of valence band (VB) (inset of Fig. 1d) [62]. Fig. 1e
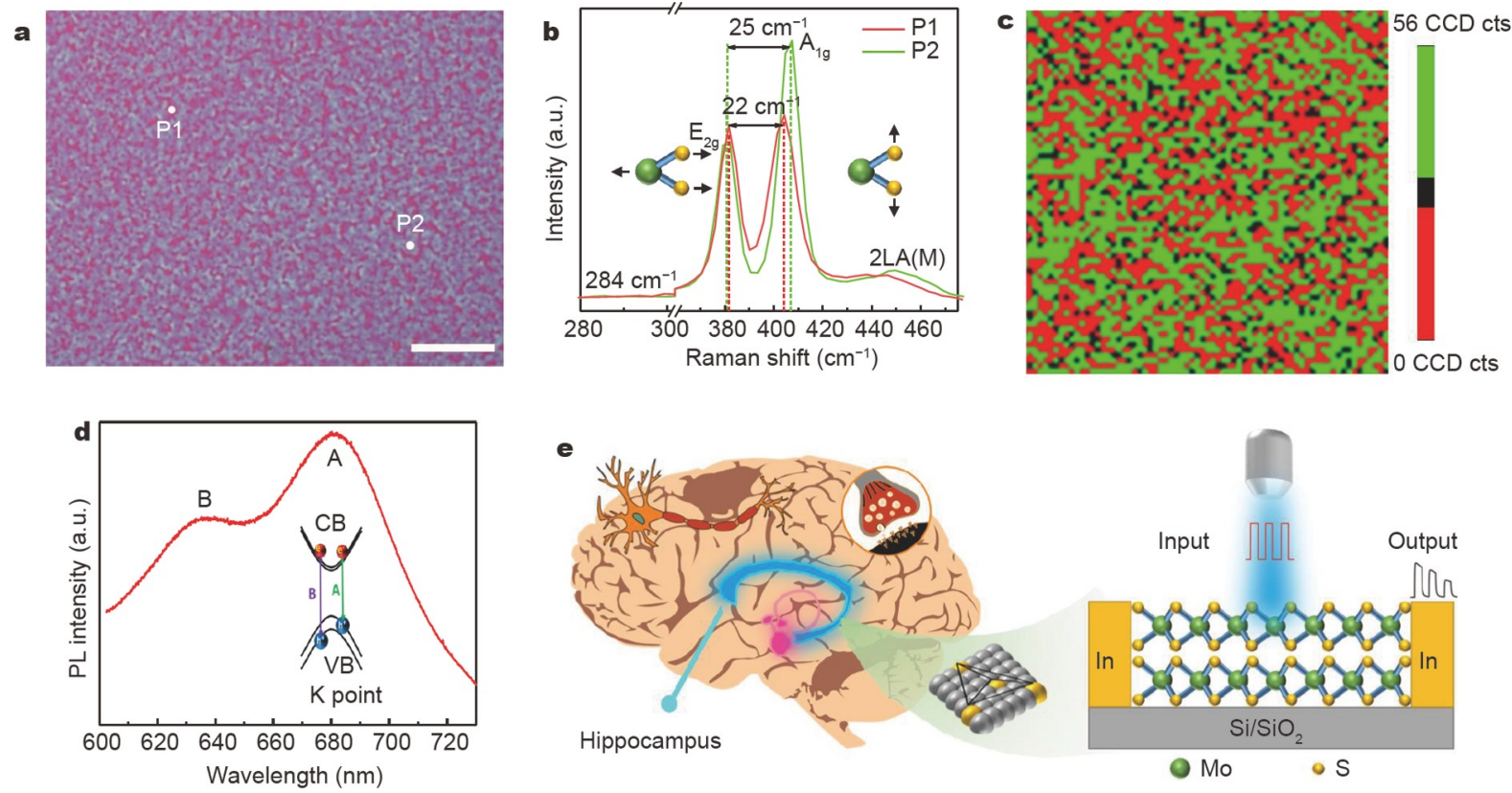

Figure 1 Material characterization results and the fabricated memory device based on $\mathrm{MoS}_{2}$ samples. (a) The optical microscope image of CVD-grown MoS samples. Scale bar: $10 \mu \mathrm{m}$. (b) The corresponding Raman spectra of marked spots (P1 and P2) in (a). (c) Raman mapping image of MoS ${ }_{2}$ at $382 \mathrm{~cm}^{-1}$. Scale bar: $3 \mu \mathrm{m}$. (d) The PL spectrum of $\mathrm{MoS}_{2}$. (e) The schematic illustration of the hippocampus-inspired memory device. 
shows the schematic structure of the neural coding memory device based on $\mathrm{MoS}_{2}$ films which is inspired by the hippocampus [63].

Fig. 2a shows the input optical stimulus (top) and the resulting output current (middle), whose on/off response tendency is similar to the memory trace [64] (bottom) of brain. According to the neurobiology, the memory formation process is accompanied by the activation and interconnection of neurons [65]. Fig. $2 b$ demonstrates the full memory process including encoding, storage and retrieval. The memory encoding and storage mainly occur in the hippocampus where the information is encoded to some specific neurons, i.e., so-called engram cells [66]. The relevant engram cells are activated (encoding, yellow ball), interconnected (storage, black line), inactivated (forgetting, grey ball) and reactivated (retrieval) during the full memory process [67]. In accordance with Ebbinghaus forgetting curve, the strength of memory decays over time $[9,68,69]$. Reasonably, the decreasing positive photocurrent and deceased reactivated photocurrent based on the $\mathrm{MoS}_{2}$ device can mimic the memory trace $[63,70]$.

The presence of structural defects in meshed $\mathrm{MoS}_{2}$ samples and the adsorbed impurities by the mesh-like surface take the responsibility for reduced carrier mobilities and conductance relaxation as charge trapping sites [71]. Especially, thermally generated traps can lead to photocurrent decay during illumination [72]. The photo-induced mechanism of decreasing positive photoconductivity with the increasing illumination time is proposed in Fig. 2c. Abundant photo-excited electron-hole pairs separate to electrodes under an applied voltage [73] resulting in sharp rising of current (stage (1) in Fig. 2c). The undesirable carrier recombination takes place due to increasing charge carriers (stage (2) in Fig. 2c) [74,75]. The recombination is known as a function of free carrier density, trap density and the probability of recombination [72]. The trap states including shallow traps and deep traps can be originated from intrinsic defects (e.g., sulfur vacancies), extrinsic charged impurities (e.g., adsorbates) or the substrate effect [43]. The carriers can be confined by these traps with corresponding short-term trapping and long-term trapping (stage (3) in Fig. 2c) [72]. The presence of recombination and trap states can affect the photoresponse. Specially, these trapped carriers can lead to a local reduction of electric field near the anode via building a depletion region [76]. This can give rise to a net decreased separation efficiency of carriers because the effective carrier mobilities are functions of the electric field [76] (stage (4) in Fig. 2c) [75]. As a result, the reduced electric field can lead to slower carrier collection efficiency by electrodes and a stronger interaction between traps and carriers [76]. Therefore, the photocurrent $\left(I_{\mathrm{ph}}\right.$, the difference of source-drain current between illumination and dark) begins to decrease after the maximal current is reached under illumination. Subsequently, an observed long-lived $I_{\mathrm{ph}}$ tail (i.e., PPC) exists when the light is switched off due to the slow charge detrapping (stage (5) in Fig. 2c). More importantly, the increasing depletion region near the anode makes the subsequent $I_{\mathrm{ph}}$ peak not exceed the last value of previous $I_{\mathrm{ph}}$ as shown in Fig. 2a (see output) [63]. The time-dependent $I_{\text {ph }}$ plot under illumination is similar to the memory trace of human under pressure. The effect of pressure on hippocampal-dependent memory has been investigated by many researchers [77]. The strength of memory is dependent on the attention intensity human pay to the stimulus [78]. More specifically, the strength of memory first increases and then deceases gradually under stress just as pressure-driven memory enhancement under transient and low-level stress and memory loss with excessive stress over time [79]. The memory retrieval shows a lower strength of memory than before due to memory decays with time [80]. This is consistent with the retrieval-extinction effect which is first discovered in Pavlovian fear conditioning [81].

Fig. 3a shows the $I_{\mathrm{ph}}$ intensity dependence on different laser power intensities under irradiation with $30 \mathrm{~s}$ light on/off cycles. The obvious decreasing positive $I_{\mathrm{ph}}$ with illumination time is observed. A power law is fitted to reveal the dependence of $I_{\mathrm{ph}}$ on laser density in Fig. 3b. The power law index of 0.785 implies the predominant bimolecular (electron-hole) recombination [72]. Fig. 3c describes the decay curve under different light intensities and the higher intensity shows the faster decay rate [75]. This is because the faster decay rate at higher light intensity can be dominated by the shallow carrier traps rather than the

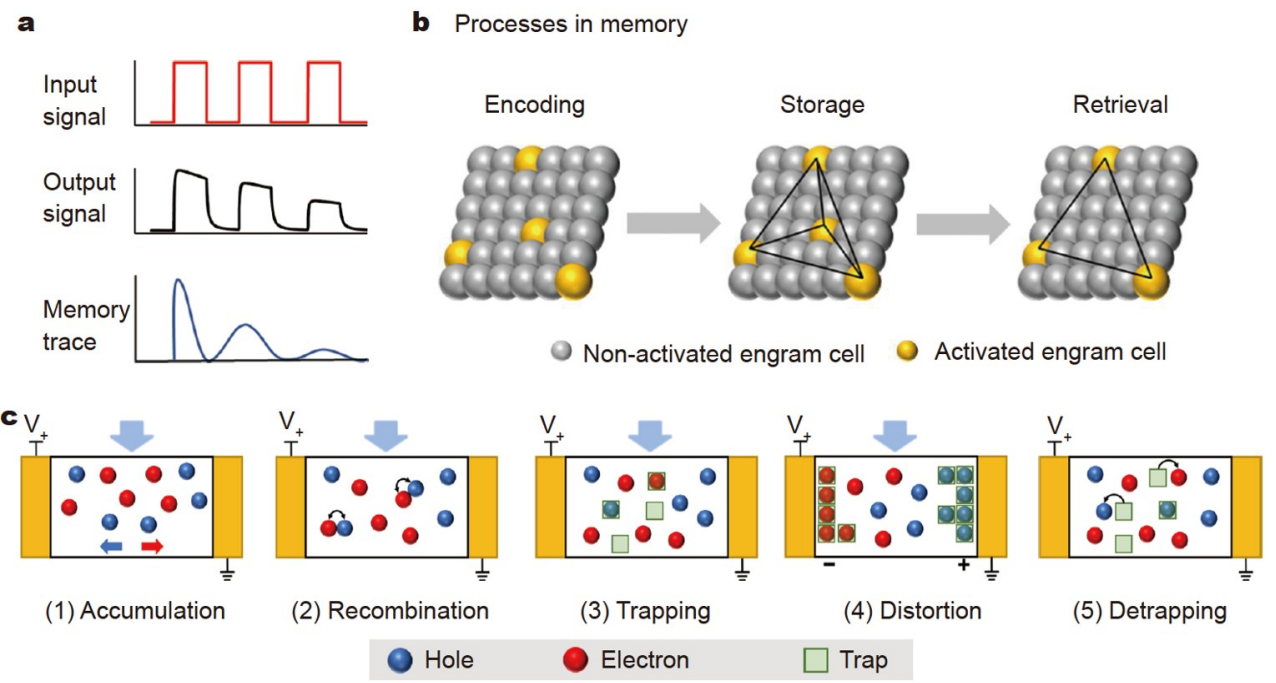

Figure 2 The memory trace process and carrier dynamics based on our memory device. (a) The input (top) and output (middle) signals of our memory devices and a typical memory trace [64] (bottom) of the human brain. (b) The schematic diagram of the full memory process including encoding, storage and retrieval. (c) The proposed various stages of carrier dynamics in the memory device. The blue arrow means the light is on. 

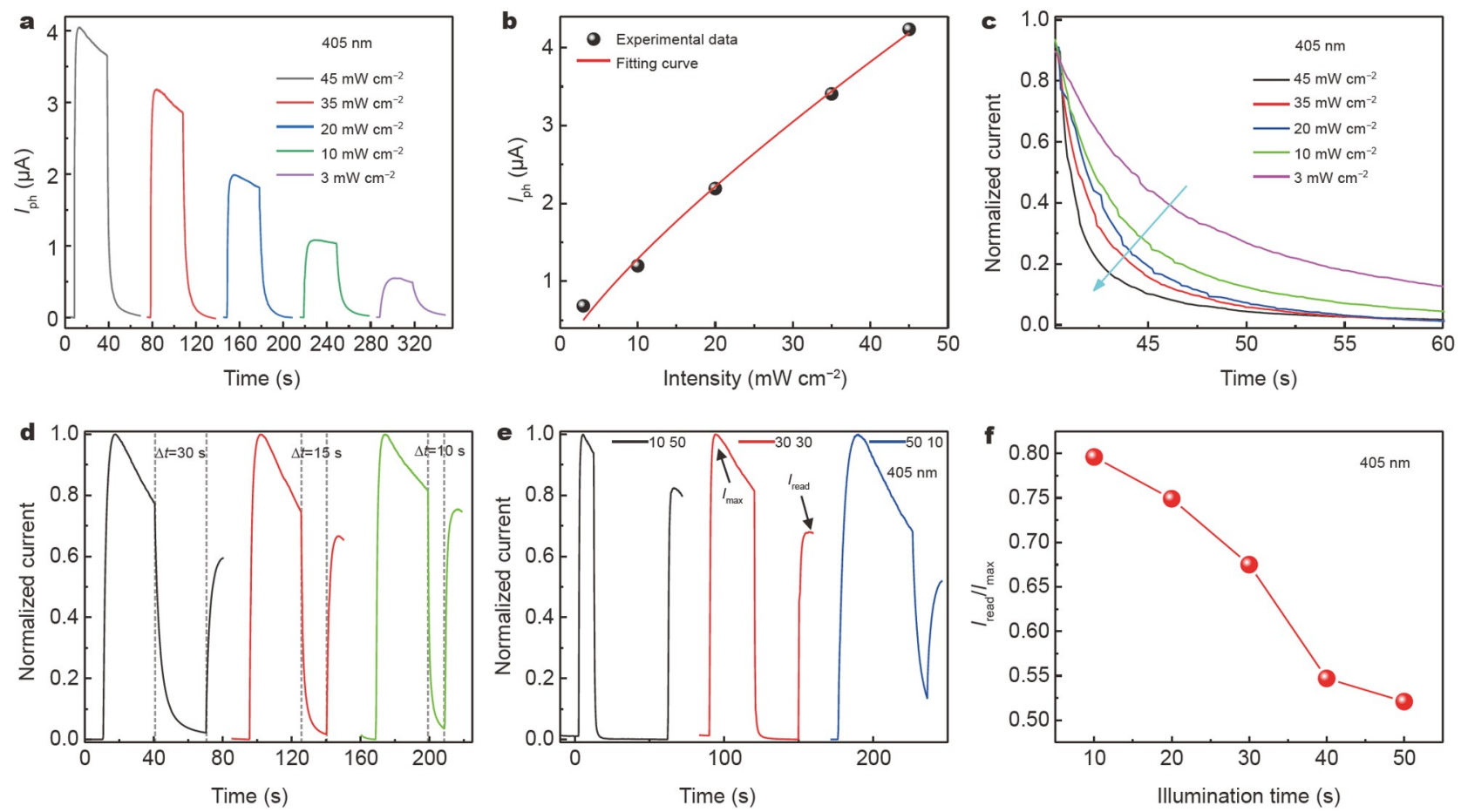

Figure 3 The photoresponse characteristics under $405 \mathrm{~nm}$ with different laser power intensities and illumination time periods. (a) Intensity-dependent $I_{\mathrm{ph}}$. (b) The relationship between $I_{\mathrm{ph}}$ and incident light intensity. (c) The decay curves of normalized $I_{\mathrm{ph}}$ under different laser power densities. (d) The normalized $I_{\mathrm{ph}}$ with different dark time periods $\Delta t$. Illumination time: $30 \mathrm{~s}$. (e) The normalized $I_{\mathrm{ph}}$ with the same period (illumination time plus dark time equals $60 \mathrm{~s}$ ). (f) The ratio of $I_{\text {read }}$ and $I_{\max }$ under different illumination times within the same $60 \mathrm{~s}$ period. The applied voltage is $2 \mathrm{~V}$.

deep carrier traps at lower light intensity [55]. The light intensity-dependent $I_{\mathrm{ph}}$ demonstrates the similar memory effect of human brain like high-intensity learning/exercise results in better memory [82]. Fig. 3d depicts the normalized current with the same illumination time and different dark time periods. The strength of memory recall decreases with the increased dark time which represents the forgetting process [83]. According to the aforementioned observations, the illumination time encoding under the same light period (i.e., one period of on/off light is $60 \mathrm{~s})$ was measured in Fig. 3e. Clearly, the information of illumination time can be inferred as shown in Fig. $3 \mathrm{f}$ via the ratio of $I_{\text {read }} / I_{\max }$, where $I_{\text {read }}$ and $I_{\max }$ means the readout $I_{\mathrm{ph}}$ on the second light activation of $10 \mathrm{~s}$ and the maximum $I_{\mathrm{ph}}$ of the first photoresponse as shown in Fig. 3e, respectively. The illumination time can be understood as the stimuli of pressure time. The stress makes human brain release relative hormones and neurotransmitter which can enhance memory [8]. However, excessive pressure may lead to memory loss and depression $[84,85]$. Thus, the decreasing positive $I_{\mathrm{ph}}$ with illumination time can mimic the pressure-induced memory behavior over time.

Encryption technology can make the encoded information more secure during the optical communication [86,87]. For our memory devices, $450 \mathrm{~nm}$ laser was used to carry the encoded information such as illumination time as demonstrated in Fig. 4a. In Fig. 4b, the 405 and $450 \mathrm{~nm}$ light excited photoresponse characteristics were obtained by illuminating the device for $30 \mathrm{~s}$, respectively. The deceasing positive $I_{\mathrm{ph}}$ is observed for both lasers. Fig. 4c, d display the illumination time and laser intensity encryption using the $450 \mathrm{~nm}$ laser, respectively. With the development of the Internet of Things (IOT) and Big Data, encryption and decryption studies for data security are sig- nificant. The encryption/decryption process is demonstrated in Fig. $4 \mathrm{e}$ using the $I_{\text {read }} / I_{\max }$ value under 405 and $450 \mathrm{~nm}$ as the key and carried new information, respectively. The encryption process is achieved by superimposing the key to the encoded information and the decryption process is realized by subtracting the key from the received information. The proposed encryption-decryption approach to illumination time can be further implemented in real-time input image applications with high key sensitivity [88]. In terms of pressure-induced memory behavior, the effect of $450 \mathrm{~nm}$ laser can be interpreted as the "stress hormone" [79] such as cortisol. The cortisol can appear when humans feel stressed or fearful [89]. The stress hormones are considered to have instant effects on focusing attention and mnemonic function [90]. The $I_{\mathrm{ph}}$ under $450 \mathrm{~nm}$ over time is also increasing and then decreasing just like the memory retention enhancement and hindering under low-level and high-level cortisol [91,92].

\section{CONCLUSIONS}

In summary, we have proposed a hippocampus-inspired device based on mesh-like $\mathrm{MoS}_{2}$ films with multilayer flakes on their surface. Based on the characteristics of the memory effect, our devices can demonstrate the pressure-triggered full memory trace over time with three independent stages of encoding, storage and retrieval. The proposed mechanism of decreasing positive $I_{\text {ph }}$ with illumination time is the electric field distortion caused by the trapped carriers predominantly. In addition, the illumination time encoding and encryption/decryption can be used for more secure optical communication [93] or as an optical encoder. Combining these two applications, the memory coding or neural coding can be implemented based on our 

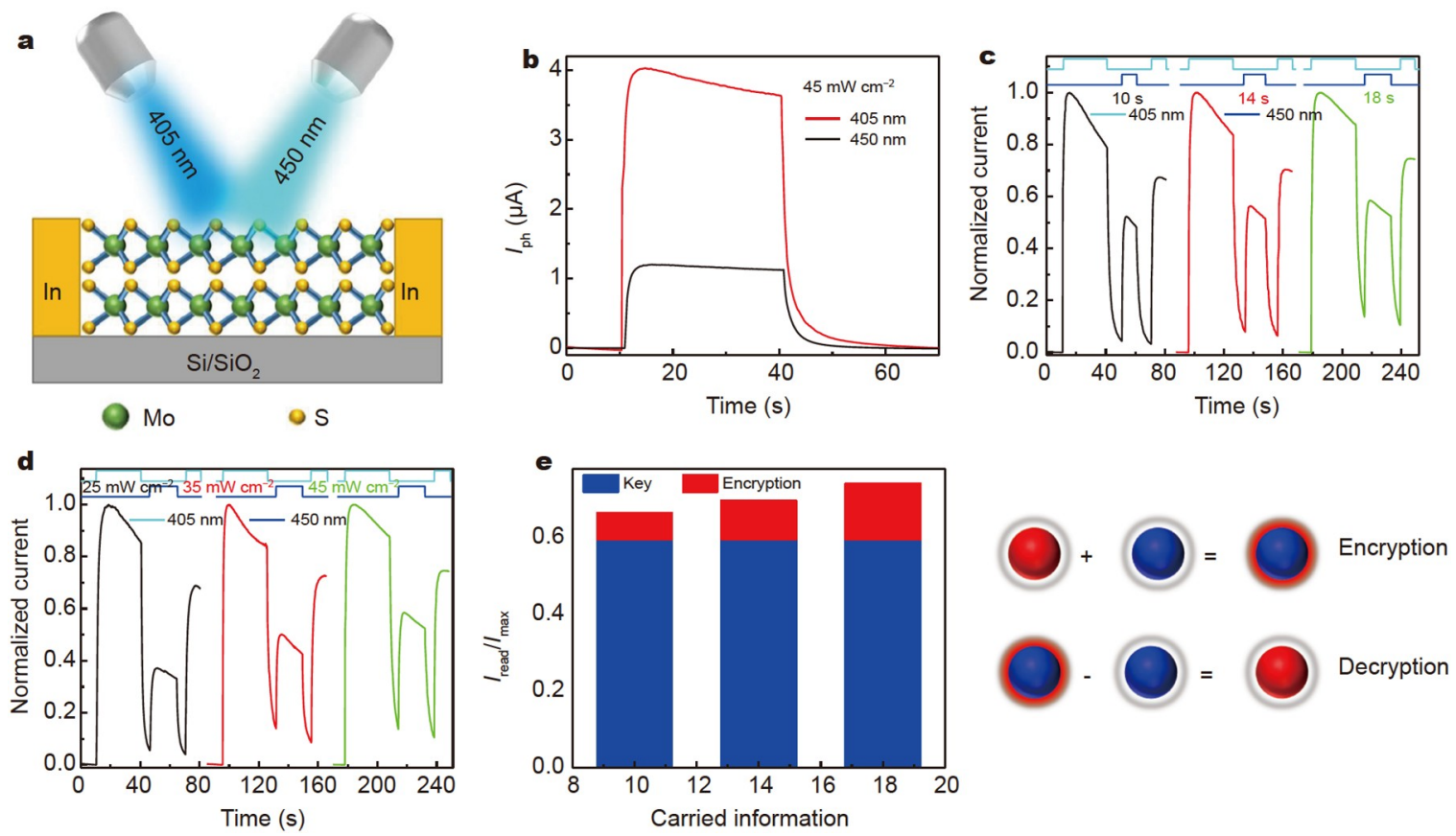

Figure 4 The photoresponse characteristics under 405 and $450 \mathrm{~nm}$ with different laser power intensities and illumination time periods. (a) The MoS 2 memory device under two lasers. (b) The $I_{\mathrm{ph}}$ under 405 and $450 \mathrm{~nm}$. Memory modulation by $450 \mathrm{~nm}$ laser with different illumination time periods (c) and laser intensities (d). (e) The graphical representation of encryption and decryption processes. The applied voltage is $2 \mathrm{~V}$.

devices. Given all this, $\mathrm{MoS}_{2}$-based optoelectronic devices provide potential applications in artificial-intelligence photosensors and evolving neural coding.

\section{Received 11 September 2021; accepted 13 October 2021; published online 26 November 2021}

1 Borst A, Theunissen FE. Information theory and neural coding. Nat Neurosci, 1999, 2: 947-957

2 Gollisch T. Throwing a glance at the neural code: Rapid information transmission in the visual system. HFSP J, 2009, 3: 36-46

3 Johnson DH, Gruner CM, Baggerly K, et al. Information-theoretic analysis of neural coding. J Comput Neurosci, 2001, 10: 47-69

4 Gupta I, Serb A, Khiat A, et al. Real-time encoding and compression of neuronal spikes by metal-oxide memristors. Nat Commun, 2016, 7: 12805

5 Eeckman FH. Computation in Neurons and Neural Systems. New York: Springer Science \& Business Media, 1994: 319

6 Tsien J. The Memory Code. Sci Am, 2007, 297: 52-59

7 Josselyn SA, Tonegawa S. Memory engrams: Recalling the past and imagining the future. Science, 2020, 367: eaaw4325

8 Mineur YS, Picciotto MR. The role of acetylcholine in negative encoding bias: Too much of a good thing? Eur J Neurosci, 2021, 53: 114125

9 Wang Y, Yin L, Huang W, et al. Optoelectronic synaptic devices for neuromorphic computing. Adv Intell Syst, 2021, 3: 2000099

10 Huang W, Xia X, Zhu C, et al. Memristive artificial synapses for neuromorphic computing. Nano-Micro Lett, 2021, 13: 85

11 Yin L, Pi X, Yang D. Silicon-based optoelectronic synaptic devices. Chin Phys B, 2020, 29: 070703

12 Tan H, Ni Z, Peng W, et al. Broadband optoelectronic synaptic devices based on silicon nanocrystals for neuromorphic computing. Nano Energy, 2018, 52: 422-430

13 Seo S, Lee JJ, Lee RG, et al. An optogenetics-inspired flexible van der Waals optoelectronic synapse and its application to a convolutional neural network. Adv Mater, 2021, 33: 2102980
14 Yin L, Huang W, Xiao R, et al. Optically stimulated synaptic devices based on the hybrid structure of silicon nanomembrane and perovskite. Nano Lett, 2020, 20: 3378-3387

15 Libby A, Buschman TJ. Rotational dynamics reduce interference between sensory and memory representations. Nat Neurosci, 2021, 24: 715-726

16 Schmidt MV, Abraham WC, Maroun M, et al. Stress-induced metaplasticity: From synapses to behavior. Neuroscience, 2013, 250: 112-120

17 Hokenson RE, Short AK, Chen Y, et al. Unexpected role of physiological estrogen in acute stress-induced memory deficits. J Neurosci, 2021, 41: 648-662

18 Wang HX, Wahlberg M, Karp A, et al. Psychosocial stress at work is associated with increased dementia risk in late life. Alzheimers Dement, 2012, 8: 114-120

19 Beltzung M, Picot C, Rempp P, et al. Investigation of the conformation of elastic chains in poly(dimethylsiloxane) networks by small-angle neutron scattering. Macromolecules, 1982, 15: 1594-1600

20 Ren A, Zou J, Lai H, et al. Direct laser-patterned MXene-perovskite image sensor arrays for visible-near infrared photodetection. Mater Horiz, 2020, 7: 1901-1911

21 Mennel L, Symonowicz J, Wachter S, et al. Ultrafast machine vision with 2D material neural network image sensors. Nature, 2020, 579: 6266

22 Huh W, Lee D, Lee CH. Memristors based on 2D materials as an artificial synapse for neuromorphic electronics. Adv Mater, 2020, 32: 2002092

$23 \mathrm{Hu}$ Y, Dai M, Feng W, et al. Monolayer hydrophilic $\mathrm{MoS}_{2}$ with strong charge trapping for atomically thin neuromorphic vision systems. Mater Horiz, 2020, 7: 3316-3324

$24 \mathrm{Li} \mathrm{C}, \mathrm{Zhu}$ J, Du W, et al. The photodetectors based on lateral monolayer $\mathrm{MoS}_{2} / \mathrm{WS}_{2}$ heterojunctions. Nanoscale Res Lett, 2021, 16: 123

$25 \mathrm{Xu} \mathrm{H}$, Zhu J, Zou G, et al. Spatially bandgap-graded $\operatorname{MoS}_{2(1-x)} \mathrm{Se}_{2 x}$ homojunctions for self-powered visible-near-infrared phototransistors. Nano-Micro Lett, 2020, 12: 26

$26 \mathrm{Xu} \mathrm{H}$, Han X, Dai X, et al. High detectivity and transparent few-layer $\mathrm{MoS}_{2}$ /glassy-graphene heterostructure photodetectors. Adv Mater, 2018, 30: 1706561 
27 Li X, Dai X, Xu H, et al. Multifunctional two-dimensional glassy graphene devices for vis-NIR photodetection and volatile organic compound sensing. Sci China Mater, 2021, 64: 1964-1976

28 Song W, Chen J, Li Z, et al. Self-powered MXene/GaN van der Waals heterojunction ultraviolet photodiodes with superhigh efficiency and stable current outputs. Adv Mater, 2021, 33: 2101059

29 Chandan , Sarkar S, Angadi B. Defects induced persistent photoconductivity in monolayer $\mathrm{MoS}_{2}$. Appl Phys Lett, 2021, 118: 172105

30 Feng X, Liu X, Ang KW. 2D photonic memristor beyond graphene: Progress and prospects. Nanophotonics, 2020, 9: 1579-1599

31 Kumar M, Abbas S, Kim J. All-oxide-based highly transparent photonic synapse for neuromorphic computing. ACS Appl Mater Interfaces, 2018, 10: 34370-34376

32 Barker Jr BG. Investigation of the Spatial Dependence of Carrier Dynamics in Semiconductor Optoelectronic Devices. Dissertation for Doctoral Degree. South Carolina: University of South Carolina, 2018

$33 \mathrm{Du}$ W, Yu P, Zhu J, et al. An ultrathin $\mathrm{MoSe}_{2}$ photodetector with nearperfect absorption. Nanotechnology, 2020, 31: 225201

$34 \mathrm{Du} \mathrm{W}, \mathrm{Li} \mathrm{C}$, Sun J, et al. Nanolasers based on 2D materials. Laser Photonics Rev, 2020, 14: 2000271

$35 \mathrm{Xu} \mathrm{H}$, Ren $\mathrm{A}, \mathrm{Wu}$ J, et al. Recent advances in 2D MXenes for photodetection. Adv Funct Mater, 2020, 30: 2000907

36 Zhu J, Xu H, Zou G, et al. $\mathrm{MoS}_{2}-\mathrm{OH}$ bilayer-mediated growth of inchsized monolayer $\mathrm{MoS}_{2}$ on arbitrary substrates. J Am Chem Soc, 2019, 141: 5392-5401

37 Wang Y, Zheng Y, Gao J, et al. Band-tailored van der Waals heterostructure for multilevel memory and artificial synapse. InfoMat, 2021, 3: $917-928$

38 Hu DC, Yang R, Jiang L, et al. Memristive synapses with photoelectric plasticity realized in $\mathrm{ZnO}_{1-x} / \mathrm{AlO}_{y}$ heterojunction. ACS Appl Mater Interfaces, 2018, 10: 6463-6470

39 Nalwa HS. A review of molybdenum disulfide $\left(\mathrm{MoS}_{2}\right)$ based photodetectors: From ultra-broadband, self-powered to flexible devices. RSC Adv, 2020, 10: 30529-30602

40 Huang Z, Zhang T, Liu J, et al. Amorphous $\mathrm{MoS}_{2}$ photodetector with ultra-broadband response. ACS Appl Electron Mater, 2019, 1: 13141321

41 Zhang Y, Li S, Li Z, et al. High-performance two-dimensional perovskite $\mathrm{Ca}_{2} \mathrm{Nb}_{3} \mathrm{O}_{10}$ UV photodetectors. Nano Lett, 2021, 21: 382-388

42 Islam MM, Dev D, Krishnaprasad A, et al. Optoelectronic synapse using monolayer $\mathrm{MoS}_{2}$ field effect transistors. Sci Rep, 2020, 10: 21870

$43 \mathrm{Wu}$ YC, Liu CH, Chen SY, et al. Extrinsic origin of persistent photoconductivity in monolayer $\mathrm{MoS}_{2}$ field effect transistors. Sci Rep, 2015, 5: 11472

44 Yu H, Wei H, Gong J, et al. Evolution of bio-inspired artificial synapses: Materials, structures, and mechanisms. Small, 2020, 17: 2000041

45 Xie D, Wei L, Xie M, et al. Photoelectric visual adaptation based on 0D$\mathrm{CsPbBr}$-quantum-dots/2D-MoS 2 mixed-dimensional heterojunction transistor. Adv Funct Mater, 2021, 31: 2010655

46 Cheng Y, Li H, Liu B, et al. Vertical 0D-perovskite/2D-MoS 2 van der Waals heterojunction phototransistor for emulating photoelectric-synergistically classical pavlovian conditioning and neural coding dynamics. Small, 2020, 16: 2005217

47 Jiang J, Hu W, Xie D, et al. 2D electric-double-layer phototransistor for photoelectronic and spatiotemporal hybrid neuromorphic integration. Nanoscale, 2019, 11: 1360-1369

48 Feng G, Jiang J, Li Y, et al. Flexible vertical photogating transistor network with an ultrashort channel for in-sensor visual nociceptor. Adv Funct Mater, 2021, 31: 2104327

49 Zhou K, Jiang J, Ding L. 2D transition metal dichalcogenides for neuromorphic vision system. J Semicond, 2021, 42: 090203

50 Gong C, Zhang $\mathrm{H}$, Wang W, et al. Band alignment of two-dimensional transition metal dichalcogenides: Application in tunnel field effect transistors. Appl Phys Lett, 2013, 103: 053513

51 Baik SS, Im S, Choi HJ. Work function tuning in two-dimensional $\mathrm{MoS}_{2}$ field-effect-transistors with graphene and titanium source-drain contacts. Sci Rep, 2017, 7: 45546

52 Chu Z, Wang CY, Quan J, et al. Unveiling defect-mediated carrier dynamics in monolayer semiconductors by spatiotemporal microwave imaging. Proc Natl Acad Sci U S A, 2020, 117: 13908-13913

$53 \mathrm{Li} \mathrm{C}$, Tong X, Yu P, et al. Carbon dioxide photo/electroreduction with cobalt. J Mater Chem A, 2019, 7: 16622-16642

54 Li C, Wang Z. An artificial biomimetic catalysis converting $\mathrm{CO}_{2}$ to green fuels. Nanoscale Res Lett, 2017, 12: 530

55 Kufer D, Konstantatos G. Highly sensitive, encapsulated $\mathrm{MoS}_{2}$ photodetector with gate controllable gain and speed. Nano Lett, 2015, 15: 7307-7313

56 Kim SY, Kwak J, Ciobanu CV, et al. Recent developments in controlled vapor-phase growth of 2D group 6 transition metal dichalcogenides. Adv Mater, 2019, 31: 1804939

57 Shi Y, Zhang H, Chang WH, et al. Synthesis and structure of twodimensional transition-metal dichalcogenides. MRS Bull, 2015, 40: 566576

58 Zhang H, Ma Y, Wan Y, et al. Measuring the refractive index of highly crystalline monolayer $\mathrm{MoS}_{2}$ with high confidence. Sci Rep, 2015, 5: 8440

59 Windom BC, Sawyer WG, Hahn DW. A Raman spectroscopic study of $\mathrm{MoS}_{2}$ and $\mathrm{MoO}_{3}$ : Applications to tribological systems. Tribol Lett, 2011, 42: $301-310$

60 Sun D, Nguyen AE, Barroso D, et al. Chemical vapor deposition growth of a periodic array of single-layer $\mathrm{MoS}_{2}$ islands via lithographic patterning of an $\mathrm{SiO}_{2} / \mathrm{Si}$ substrate. 2D Mater, 2015, 2: 045014

61 Lin Z, Zhao Y, Zhou C, et al. Controllable growth of large-size crystalline $\mathrm{MoS}_{2}$ and resist-free transfer assisted with a $\mathrm{Cu}$ thin film. Sci Rep, 2015, 5: 18596

62 Golovynskyi S, Irfan I, Bosi M, et al. Exciton and trion in few-layer $\mathrm{MoS}_{2}$ : Thickness- and temperature-dependent photoluminescence. Appl Surf Sci, 2020, 515: 146033

63 Belisle RA, Nguyen WH, Bowring AR, et al. Interpretation of inverted photocurrent transients in organic lead halide perovskite solar cells: Proof of the field screening by mobile ions and determination of the space charge layer widths. Energy Environ Sci, 2017, 10: 192-204

64 Bernacchia A, Seo H, Lee D, et al. A reservoir of time constants for memory traces in cortical neurons. Nat Neurosci, 2011, 14: 366-372

65 Poo MM, Pignatelli M, Ryan TJ, et al. What is memory? The present state of the engram. BMC Biol, 2016, 14: 40

66 Wang $\mathrm{C}$, Yue $\mathrm{H}, \mathrm{Hu} \mathrm{Z}$, et al. Microglia mediate forgetting via complement-dependent synaptic elimination. Science, 2020, 367: 688-694

67 Tonegawa S, Morrissey MD, Kitamura T. The role of engram cells in the systems consolidation of memory. Nat Rev Neurosci, 2018, 19: 485498

68 Laborieux A, Ernoult M, Hirtzlin T, et al. Synaptic metaplasticity in binarized neural networks. Nat Commun, 2021, 12: 2549

69 Ebbinghaus H. Uber das Gedachtnis. Untersuchungen zur experimentellen Psychologie. Leipzig: Duncker \& Humblot, 1885: 185

$70 \mathrm{He} \mathrm{Z}$, Shen $\mathrm{H}$, Ye D, et al. An organic transistor with light intensitydependent active photoadaptation. Nat Electron, 2021, 4: 522-529

71 Durand C, Zhang X, Fowlkes J, et al. Defect-mediated transport and electronic irradiation effect in individual domains of CVD-grown monolayer $\mathrm{MoS}_{2}$. J Vac Sci Technol B, 2015, 33: 02B110

72 Basumatary P, Agarwal P. Photocurrent transient measurements in $\mathrm{MAPbI}_{3}$ thin films. J Mater Sci-Mater Electron, 2020, 31: 10047-10054

73 Gong Y, Lei S, Ye G, et al. Two-step growth of two-dimensional WSe $e_{2}$ $\mathrm{MoSe}_{2}$ heterostructures. Nano Lett, 2015, 15: 6135-6141

74 Daboczi M, Kim J, Lee J, et al. Towards efficient integrated perovskite/ organic bulk heterojunction solar cells: Interfacial energetic requirement to reduce charge carrier recombination losses. Adv Funct Mater, 2020, 30: 2001482

75 McNeill CR, Hwang I, Greenham NC. Photocurrent transients in allpolymer solar cells: Trapping and detrapping effects. J Appl Phys, 2009, 106: 024507

76 Musiienko A, Pipek J, Praus P, et al. Deciphering the effect of traps on electronic charge transport properties of methylammonium lead tribromide perovskite. Sci Adv, 2020, 6: eabb6393

77 Cohen N, Kinney KS. PROLOGUE: Exploring the Phylogenetic History of Neural-immune System Interactions: An Update. In: Robert Ader. Psychoneuroimmunology (Fourth Edition). Burlington: Academic Press, 2007: 1-38 
78 Asok A, Leroy F, Rayman JB, et al. Molecular mechanisms of the memory trace. Trends Neuroscis, 2019, 42: 14-22

79 Hoscheidt SM, LaBar KS, Ryan L, et al. Encoding negative events under stress: High subjective arousal is related to accurate emotional memory despite misinformation exposure. Neurobiol Learn Mem, 2014, 112: 237-247

80 Cowan $\mathrm{N}$. What are the differences between long-term, short-term, and working memory? Prog Brain Res, 2008, 169: 323-338.

81 Liu J, Zhao L, Xue Y, et al. An unconditioned stimulus retrieval extinction procedure to prevent the return of fear memory. Biol Psychiatry, 2014, 76: 895-901

82 Frith E, Sng E, Loprinzi PD. Randomized controlled trial evaluating the temporal effects of high-intensity exercise on learning, short-term and long-term memory, and prospective memory. Eur J Neurosci, 2017, 46: 2557-2564

83 Chen T, Wang X, Hao D, et al. Photonic synapses with ultra-low energy consumption based on vertical organic field-effect transistors. Adv Opt Mater, 2021, 9: 2002030

84 de Quervain D, Schwabe L, Roozendaal B. Stress, glucocorticoids and memory: Implications for treating fear-related disorders. Nat Rev Neurosci, 2017, 18: 7-19

85 Tye KM, Mirzabekov JJ, Warden MR, et al. Dopamine neurons modulate neural encoding and expression of depression-related behaviour. Nature, 2013, 493: 537-541

86 Refregier P, Javidi B. Optical image encryption based on input plane and Fourier plane random encoding. Opt Lett, 1995, 20: 767-769

87 Ren $\mathrm{A}$, Wang $\mathrm{H}$, Zhang W, et al. Emerging light-emitting diodes for next-generation data communications. Nat Electron, 2021, 4: 559-572

88 Yasser I, Mohamed MA, Samra AS, et al. A chaotic-based encryption/ decryption framework for secure multimedia communications. Entropy, 2020, 22: 1253

89 Schulz P, Kirschbaum C, Prüßner J, et al. Increased free cortisol secretion after awakening in chronically stressed individuals due to work overload. Stress Med, 1998, 14: 91-97

90 Henckens MJAG, Hermans EJ, Pu Z, et al. Stressed memories: How acute stress affects memory formation in humans. J Neurosci, 2009, 29: 10111-10119

91 Lupien SJ, de Leon M, de Santi S, et al. Cortisol levels during human aging predict hippocampal atrophy and memory deficits. Nat Neurosci, 1998, 1: 69-73

92 Kim JJ, Diamond DM. The stressed hippocampus, synaptic plasticity and lost memories. Nat Rev Neurosci, 2002, 3: 453-462

93 Li J, Yu P, Cheng $\mathrm{H}$, et al. Optical polarization encoding using grapheme-loaded plasmonic metasurfaces. Adv Opt Mater, 2016, 4: 91-98

Acknowledgements This work was supported by the Innovation Group Project of Sichuan Province (20CXTD0090), the Fundamental Research Funds for the Central Universities (ZYGX2019Z018), the University of Electronic Science and Technology of China (UESTC) Shared Research Facilities of Electromagnetic Wave and Matter Interaction (Y0301901290100201), the National Natural Science Foundation of China (62004025), the International Postdoctoral Exchange Fellowship Program (Talent-Introduction Program, 244125), the UESTC 100-Talent Project Fund, and China Postdoctoral Science Foundation (244125).

Author contributions $\mathrm{Li} \mathrm{C}, \mathrm{Du} \mathrm{W}$ and $\mathrm{Wu}$ J conceived and designed the research project. Li C synthesized the samples and performed the optical spectroscopy. Li C fabricated the device and carried out the optoelectronic measurements with the help of Du W. Liu H participated in setting up the measurements. Li C analyzed the data and wrote the manuscript with the contribution from Yang M. Xu H, Wu J and Wang Z supervised the project and revised the manuscript. All authors contributed to the general discussion. Conflict of interest The authors declare that they have no conflict of interest.

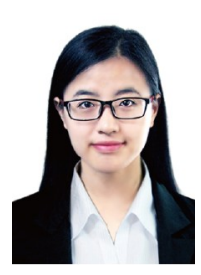

Caihong $\mathrm{Li}$ received her BS degree in microelectronics and solid-state electronics from the School of Electronic information, Qingdao University in 2016. She is currently a $\mathrm{PhD}$ candidate of microelectronics and solid-state electronics at the University of Electronic Science and Technology of China (UESTC) under the supervision of Prof Jiang $\mathrm{Wu}$. Her research interests include the preparation and application of $2 \mathrm{D}$ optoelectronic materials.

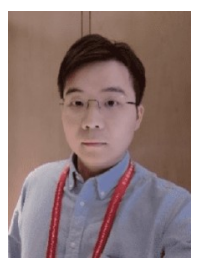

Hao Xu is a research professor at UESTC. He received his Master's degree in microelectronics from Shanghai Institute of Microsystem and Information Technology, Chinese Academy of Sciences, and his $\mathrm{PhD}$ degree in photonics and nanotechnology from the University College London (UCL). His current research is focused on low-dimensional semiconductors and their applications for nanoelectronics and optoelectronics.

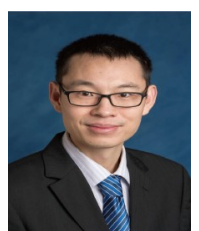

Jiang Wu obtained his $\mathrm{PhD}$ degree from the University of Arkansas Fayetteville in 2011. After receiving his $\mathrm{PhD}$ degree, he joined UESTC as an associate professor and later professor. He joined UCL as a research associate in 2012 and then lecturer in the Department of Electronic and Electrical Engineering at UCL from 2015 to 2018. He is now a professor at UESTC. He is also a Fellow of the Higher Education Academy. His research interests include optoelectronic applications of semiconductor heterostructures.

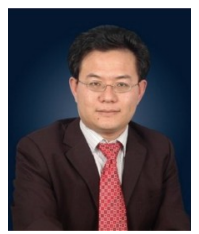

Zhiming Wang received his BS degree in applied physics from Qingdao University, Qingdao, China in 1992, his MS degree in physics from Beijing University in Beijing, China in 1995, and his PhD degree in condensed matter physics from the Institute of Semiconductors, Chinese Academy of Sciences in Beijing, China in 1998. He is now a professor, working at UESTC. His research centers on the optoelectronic properties of low-dimensional semiconductor nanostructures and corresponding applications in photovoltaic devices.

\section{海马体启发的与光照时长相关的记忆器件用于神经 编码}

李彩虹 ${ }^{1}$, 杜文 ${ }^{1}$, 刘和桩 $^{1}$, 杨茂 ${ }^{2}$, 徐浩 $2,3^{*}$, 巫江 ${ }^{1,3^{*}}$, 王志明 $1^{*}$

摘要 本文在海马体的启发下设计了一种与光照时长相关的记忆器件. 与常规的持续性正/负电导不同, 基于类网状二硫化锄沟道层的记忆器 件表现出随光照时长逐渐降低的正光电导. 由于光照下缺陷诱导的捕 获陷阱会在阳极附近形成耗尽层, 导致局部电场畸变, 从而使载流子分 离效率净降低, 该器件表现出随光照时长先增加后降低的光响应, 而且 再次光激发的响应电流低于前一次光激发的电流, 即再次光激发读出 电流与前一次光照时长相关. 因此在 $405 \mathrm{~nm}$ 激光光照下该器件可用于 光照时长编码和模拟压力诱导下的完整记忆行为包括记忆编码、存储 和再检索. 此外, 通过再引入 $450 \mathrm{~nm}$ 激光可以实现光照时长编码的加密 与解密, 同时也可以进一步模拟压力引发的相应激素对记忆的影响. 